\title{
Theories for multiple resonances
}

\author{
D. Klakow, M. Weber, and P.-G. Reinhard \\ Institute für Theoretische Physik II, Staudtstr. 7 \\ Universität Erlangen-Nürnberg, D-91058 Erlangen, Germany
}

August 6, 2018

\begin{abstract}
Two microscopic theories for multiple resonances in nuclei are compared, n-particle-hole RPA and quantized Time-Dependent Hartree-Fock (TDHF). The Lipkin-Meshkov-Glick model is used as test case. We find that quantized TDHF is superior in many respects, except for very small systems.
\end{abstract}

\section{Introduction}

The most prominent excitations of the nucleus are the giant resonances. One has therefore dreamed about multiple excitation of giant resonances quite early in the development of nuclear theory [1]. Recent experimental progress has brought those multiple resonances into the range of observability [2]. This has inspired several

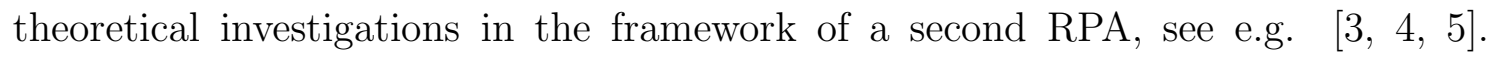
Multiple excitations of basic collective modes are well known from the low energyspectra of soft nuclei. They are most often characterized phenomenologically in terms of interacting elementary Bosons, e.g. surface vibrations and pairing modes [6]. Theoretical foundations for such a collective description of the low-energy modes have been long searched for in the framework of the Boson expansions, for a review see [7]. This task has turned out to be very involved, and a possible explanation is that the low energy modes are far from a simple picture of collective motion as mere 
shape vibrations of the mean field. Motion along a deformation path is accompanied by dramatic reoccupations amongst crossing single particle levels [8] having possibly curious consequences for the collective dynamics [9]. Giant resonances, on the other hand, represent the much cleaner collective modes, dominated by the mean field and without noticeable pairing effects. The theoretical appeal of the multiple resonances is then to study large amplitude motion in a case where the underlying elementary Boson is fairly well understood. The basic modes, the giant resonances, are usually described within the RPA, the theory of small $1 p h$ excitation about the Hartree-Fock ground state [10]. Multiple resonances require extensions beyond standard RPA. A straightforward next step is the "second RPA" extending the treatment to the space of 2-ph excitations [3, 5]. Boson expansions, which have also been considered in that context [4] confine the extension to the collective modes but allow to go further in the anharmonic terms. Somewhat more general are the "higher RPA" approaches considering coherent mixtures of $n$-ph-excitations. The equations-ofmotion techniques as outlined in [10, 11] provide an extremely useful tool to derive those expansions along a given set of $n$-ph operators. A much different point of view is presented by Time-Dependent Hartree-Fock (TDHF) approach which pronounces more the vibrating mean field. Its small amplitude limit delivers also the RPA. The spectra for higher modes can be computed with quantized TDHF which picks the quantum states amongst all the classically allowed energies by requiring strictly periodic TDHF orbit which have integer action along one closed orbit [12]. It is the aim of this paper to compare the results on multiple resonances as computed with quantized TDHF or with an $n$-ph-RPA derived from an equations of motion technique. The comparison is performed in a schematic model for nuclear dynamics in the active shell around the Fermi surface, the Lipkin-Meshkov-Glick (LMG) model 13, 14. We will use in the following the abbreviation RPA- $N$ for such a higher RPA which includes up to $N$-ph excitations.

The paper is outlined as follows: In section 2, we introduce briefly the LMG model. In section 3, we recapitulate the equations-of-motion technique and work it out for the present model. In section $₫$, we explain quantized TDHF and how we compute it. Finally in section 5, we present and discuss the results from both schemes. 


\section{The Lipkin Model}

The LMG model [13, 14] is a simplified shell model with two degenerate bands of single Fermion states $a_{s, m}^{\dagger}, s \in\{+,-\}, m \in[1 \ldots \Omega]$. The uncoupled ground state of the model carries all states $s=-$ as occupied and $s=+$ as empty. Due to the degeneracy, all single particle excitation energies are the same $\epsilon$. The model Hamiltonian reads

$$
H=\epsilon K_{0}+\chi \frac{\epsilon}{2(\Omega-1)}\left(K_{+} K_{+}+K_{-} K_{-}\right)
$$

where the basic operators are

$$
\begin{aligned}
K_{0} & =\sum_{m=1 \ldots \Omega}\left[a_{+, m}^{\dagger} a_{+, m}-a_{-, m}^{\dagger} a_{-, m}\right] \\
K_{+} & =\sum_{m=1 \ldots \Omega} a_{+, m}^{\dagger} a_{-, m} \\
K_{-} & =K_{+}^{\dagger}
\end{aligned}
$$

The second term, quadratic in the $K_{ \pm}$, models the residual interaction. The $K_{ \pm}$are $1 p h$ operators with respect the uncoupled ground state and thus the Hamiltonian (四) is tailored for model studies of the dynamics along the $1 p h$ channel, i.e. for mean field dynamics. The dimensionless parameter $\chi$ describes the interaction strength to the single $=$ particle splitting $\epsilon$. The scaling with $1 /(\Omega-1)$ places the critical coupling at $\chi=1$ almost independent of the system size $\Omega$.

Note the two important features of the LMG model: First, the residual interaction is only active for vertical $1 p h$ excitations, i.e. those which leave the secondary quantum number $m$ invariant. Second, all substates $m$ are handled with the same weight and phase in each of the three operators $K_{\alpha}$. This introduces a high symmetry in the model such that we have an exactly decoupling collective subspace which is spanned by the Thouless transformations $|\Phi\rangle=\exp \left(i \sum_{\alpha \in\{-, 0,+\}} c_{\alpha} K_{\alpha}\right)|0\rangle$ with the basic collective operators $K_{\alpha}$ [15. Moreover, these basis operators form a simple $S U(2)$ algebra

$$
\left[K_{0}, K_{ \pm}\right]= \pm K_{ \pm} \quad \text { and } \quad\left[K_{+}, K_{-}\right]=2 K_{0}
$$

All further evaluations, in the equations-of-motion technique as well as in quantized TDHF, require only this algebra and, of course, the model Hamiltonian (11). 
In spite of its simplicity, the LMG model unfolds a rich variety of scenarios for the collective motion, nearly harmonic motion for small $\chi$, increasing anharmonicities with increasing $\chi$, and unstable motion with subsequent phase transition near $\chi=1$, for a visualization see the beginning of section 5. It provides the ideal testing ground for our purposes. In fact, it was one of the originals aims of the LMG model to understand the appearance of collective modes in the RPA. It is the mode which is excited by a mix $x K_{+}-y K_{-}$and whose frequency is $\omega=\epsilon \sqrt{1-\chi^{2}}$ in the domain $\chi<1$.

\section{Equations-of-motion and RPA- $N$}

The equations-of-motion technique as outlined in [11, 10] is in our opinion the most obvious way to formulate a RPA- $N$ as an algebra of selected excitation operators. The aim is to optimize a set of excitations operators $Q_{\nu}^{\dagger}$ which excite the state

$$
|\nu\rangle=Q_{\nu}^{\dagger}|0\rangle
$$

out of a correlated ground state $|0\rangle$. The excitation operators are taken as linear superposition from a basis set of operators $\mathcal{Q}_{\alpha}$

$$
Q_{\nu}=\sum_{\alpha} c_{\alpha}^{\nu} \mathcal{Q}_{\alpha}
$$

In the ideal case, the excitation operators should fulfill he Heisenberg equation

$$
\left[H, Q_{\nu}^{\dagger}\right]=\left(E_{\nu}-E_{0}\right) Q_{\nu}^{\dagger}
$$

But this can rarely be fulfilled in practice because the algebra of the $\mathcal{Q}_{\alpha}$ and $H$ is not closed. One thus requires Eq. (17) in the average after "stabilizing" the expression to a double commutator. This leads to the variational equations

$$
<0\left|\left[\delta Q,\left[H, Q_{\nu}^{\dagger}\right]\right]\right| 0>=\omega_{\nu}<0\left|\left[\delta Q, Q_{\nu}^{\dagger}\right]\right| \nu>\quad, \quad \omega_{\nu}=\left(E_{\nu}-E_{0}\right)
$$

where $\delta Q$ stands for any variation in the given space of $\mathcal{Q}_{\alpha}$. The correlated ground state $|0\rangle$ can be generated with a similar variational equation. It is written in the form

$$
\left|0>=e^{S}\right| H F>
$$


where $S$ is an antihermitian operator and $\mid H F>$ the Hartree-Fock ground state. The Operator $S$ is determined by [1]

$$
<0|[\delta S, H]| 0>=0
$$

where $\delta S$ is again any variation in the given basis space of operators.

The spectrum of collective excitations in the Lipkin Model is generated from the basis states $K_{+}^{n}$ and $K_{-}^{n}$. We thus make for the excitation operator the ansatz

$$
Q_{\nu}^{\dagger}=\sum_{n=1}^{N} x_{n, \nu} K_{+}^{n}-y_{n, \nu} K_{-}^{n}
$$

and similarly for the correlation operator

$$
S=\sum_{n=1}^{M} \gamma_{n, \nu}\left(K_{+}^{n}-K_{-}^{n}\right)
$$

The $N$ and $M$ represent the order of the expansion, in practice the maximal order of $N$-ph or $M$-ph operator taken into account. The equations-of-motion (8) and (10) constitute a set of equations to determine $\omega_{\nu}, x_{i, \nu}, y_{i, \nu}(n=1 . . N, \nu=1 . . N)$ and $\gamma_{n}(n=1 . . M)$. It is most consistent to use the same order $M \approx N$ in both pieces and this yields usually the best results for a given expense. An exception is the conventional RPA which appears as the case $N=1$ and $M=0$ within that scheme. The enhancement to $M=1$ is unnecessary in that case because the $1 p h$ states decouple from the Hartree-Fock ground state [16]. For any other $N>1$, it is best to chose $N=M$ and this is what we call the higher RPA of order $N$, in short RPA- $N$. We will consider in the following the two particular examples RPA-2 and RPA-5.

\section{Quantized TDHF}

TDHF approximates the dynamics of a many-Fermion system by a time-dependent Slater state $|\Phi(t)\rangle$. The time-evolution of $|\Phi(t)\rangle$ is optimized by deriving the TDHFequations from the variational principle of stationary action

$$
\delta \int<\Phi\left|i \hbar \frac{d}{d t}-H\right| \Phi>d t=0
$$


where $\delta$ denotes variations within the subspace of Slater states. TDHF delivers a deterministic equation-of-motion for $|\Phi(t)\rangle$. It can be interpreted as representing the classical limit of many-Fermion dynamics. The interpretation is pertinent in particular with respect to the fact that TDHF motion is possible for any initial condition $|\Phi(0)\rangle$ at any energy. However, it can be complemented by a (semiclassical) quantization, as has been shown by very different approaches, as e.g. the functional integral representation of the Green function $G(E)=\operatorname{tr} \frac{1}{\hat{H}-E}$ [17, 18, 19] or the method of gauge invariant periodic quantization [20, 21]. The quantum states are those solutions of the TDHF equation (13) which fulfill two additional conditions: First, they are strictly periodic including the phase,

$$
|\Phi(t+T)\rangle=|\Phi(t)\rangle
$$

and second, they fulfill the quantization condition

$$
\int_{0}^{T}<\Phi\left|i \hbar \frac{d}{d t}\right| \Phi>=2 n \pi \hbar
$$

where $n$ is some integer number.

The most problematic part of quantized TDHF is to find periodic solutions 22 in the chaotic manifold of trajectories of a realistic TDHF calculation. Fortunately, this is no problem at all in the LMG model because the motion of interest is reduced to the one collective degree-of-freedom spanned by the $K_{ \pm}$as defined in section 2 . First tests of quantized TDHF have therefore been performed within this model [17, 20]. According to the Thouless theorem, each Slater determinant of a given system can be generated from a reference determinant $\left|\Phi_{0}\right\rangle$ as $|\Phi\rangle=\exp \left(A_{p h}\right)\left|\Phi_{0}\right\rangle$ where $A_{p h}$ is a $1 p h$ operator. We aim to span the space of "collective deformations" outgoing from the Hartree-Fock ground state. This is achieved by the coherent states

$$
\left|z>=e^{z K_{+}}\right| H F>\left(1+|z|^{2}\right)^{-\frac{\Omega}{2}}
$$

which are labelled by the complex shift parameter $z$. Note that these coherent states are already normalized.

The expectation values which are necessary for Eq. (13) are easily evaluated

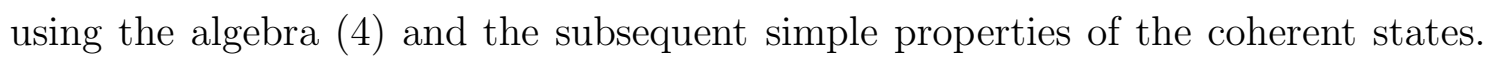


We obtain

$$
\begin{aligned}
<z\left|i \hbar \frac{d}{d t}\right| z> & =-\frac{\Omega}{2} \hbar(1-\cos \psi) \dot{\phi} \\
<z|H| z> & =-\frac{\Omega \epsilon}{2}\left(\cos \psi-\frac{\chi}{2} \sin ^{2} \psi \cos 2 \phi\right) \\
z & =e^{i \phi} \tan \left(\frac{\psi}{2}\right)
\end{aligned}
$$

The time evolution of the coherent state is contained in the time-evolution of the parameter $z$. By Variation according to (13) we find the TDHF equations as equationsof-motion for $z$

$$
\begin{aligned}
\frac{\hbar}{\epsilon} \dot{\psi} & =-\chi \sin \psi \sin 2 \phi \\
\frac{\hbar}{\epsilon} \dot{\phi} & =-(\chi \cos \psi \cos 2 \phi+1)
\end{aligned}
$$

The periodicity condition (14) is trivially fulfilled because we have an effectively two-dimensional phase space. It remains to watch the quantization condition (15) which reads in the LMG model

$$
-\frac{\Omega}{2} \int_{0}^{T}(1-\cos \psi) \dot{\phi} d t=-\frac{\Omega}{2} \oint(1-\cos \psi) d \phi=2 n \pi
$$

The initial condition of the coupled differential equations (20) and (21) are varied until the solution fulfills the quantization condition (22). The (quantum) excitation energy is then provided by the expectation value (18) which is constant along the stationary path.

\section{Results}

The LMG model has essentially two parameters, namely $\chi$, the relative coupling strength, and $\Omega$, the particle number or size of active phase space. Variation of $\chi$ varies the ground state deformation and the anharmonicity of the collective resonances. The uncoupled ground state of the model is the Hartree-Fock ground state for $\chi<1$ whereas a transition to a "deformed" state occurs for $\chi>1$. Close to harmonic excitations appear at $\chi \ll 1$ and $\chi \gg 1$ whereas increasing anharmonicities build up if the critical point $\chi=1$ is approached from both sides. Variation of $\Omega$ 
influences the softness of the transition around the critical $\chi=1$, being softer for small $\Omega$ and developing a sharp phase transition for $\Omega \longrightarrow \infty$. In order to visualize the dynamics of the system, we show in Fig. 1 the deformation energies versus collective deformation angle $\psi$.

The case $\chi=0.4$ displays a nearly parabolic potential indicating that we have to expect essentially harmonic motion there with small anharmonic perturbations. The case $\chi=1$ shows an extremely soft and anharmonic potential. The system is in a critical regime where small perturbations lead to huge reactions. The effect remains finite for finite $\Omega$ and tends to critical, i.e. infinite, fluctuations for $\Omega \longrightarrow \infty$. The case $\chi=4$ shows again a well developed ground state with small oscillations about it in the first excited state. But note that this ground state is placed at finite deformation $\psi$ and that it appears twice at exactly the same energy. As a consequence, we will have a doubled excitation spectrum because each mode has a symmetric copy about the other ground state. The excitation energies from quantized TDHF or RPA- $N$ are exactly degenerate. The degeneracy of the exact excited states is removed a little bit due to tunneling processes through the barrier at $\psi=0$. None of the presently discussed theories can reproduce this splitting.

In Fig. 2, we compare the results of quantized TDHF, RPA-5 and RPA-2 with the exact solution up the fourth resonance excitation. The figure concentrates on the regime of undercritical couplings. Three system sizes are considered which are comparable in the nuclear case to the phase spaces of light, medium and heavy nuclei. The first excited state is very well described by all methods. The tiny differences seen there would favour quantized TDHF and, somewhat surprisingly, RPA-5 looks a bit inferior to RPA-2 for small $\Omega$. But one should not overstress these details. The comforting message is that plain RPA performs well for the first excited state in the regime of undercritical coupling. More visible differences show up with increasing excitations. Quantized TDHF looks inferior for the small system size $\Omega=5$ where RPA-5 performs very well. The reverse happens for medium and large systems where the result from quantized TDHF are astonishingly close to the exact solution. This complies with the interpretation of TDHF as a classical limit of the many-Fermion dynamics. This is a classical limit in the sense of a $1 / \Omega$ expansion and it becomes increasingly valid with increasing system size, here $\Omega$. On the other 
hand, the success of the RPA- 5 for $\Omega=5$ is not so surprising because that expansion is then close to complete (note that it is not yet fully complete at 5 th order because the 0 -ph operator $K_{0}$ is missing in the expansion and has to be regenerated from pairs of $K_{ \pm}$). Finally, we note that the RPA-2 can by definition only describe the system up to the second excited state. But it performs surprisingly well at its upper limit $\omega_{2}$ and can reliably be used instead of RPA-5 there.

In Fig. 3, we show the same comparison for a wider range of couplings $\chi$. The exact spectra reflect nicely the appearance of two equivalent ground states in that pairs of excited states develop for $\chi \longrightarrow \infty$. The first excited state at $\chi<1$ goes to zero excitation and thus merges into one of the two ground states of the system, in fact it represents the antisymmetric combination whereas the former ground state develops into the symmetric combination. The transition is best developed for the large $\Omega$ whereas the small $\Omega=5$ stays far from the asymptotic stage within the range of $\chi$ considered. It is interesting to see that the different approximations handle the transitional region much differently. The plain RPA is known to break down near the critical $\chi$ [23]. This feature persists also for the higher $\operatorname{RPA}-N$. Even the RPA-5 runs into difficulties if $\chi$ grows near 1 . This region has to be excluded from any RPA-like expansion. It is a typical application case for theories of large amplitude collective motion, as e.g. the generator-coordinate method [23]. RPA becomes applicable again beyond the transition where well bound ground state minima develop again, see Fig. 1. Accordingly, a new branch of RPA solutions can be computed. We show in Fig. 3 the results from the RPA-2 because we have there at most a second excited state and we know from Fig. 2 that this level of approximation is fully sufficient up the second excitation. The RPA solutions in the regime of large $\chi$ look fair for large $\chi$ but run also in insurmountable difficulties if $\chi$ is lowered towards the critical $\chi=1$. We thus have a separate branch of RPA solutions for large $\chi$ which is disconnected from the branch of RPA solutions in the regime of small couplings. As expected from Fig. 1, the motion in the deformed minima is much less harmonic. As a consequence, the RPA-2 gives less reliable results in the regime $\chi>1$, in particular for the small system. For example, it misses the coupling through the barrier and gives always exactly degenerate pairs of excitation states.

It is interesting to see that quantized TDHF is much more robust at the critical 
point and provides surprisingly appropriate results there. It is indeed able to stretch branches of solutions over all ranges of the coupling $\chi$. However, a bit of care is still required because once in a while a branch changes discontinously. This happens in the strong coupling regime whenever the quantized energy crosses the barrier between the left and the right minimum. TDHF as a classical theory cannot describe tunneling through the barrier. Thus the branch of extended motion over the barrier ceases to exist and two new branches below the barrier develop in the separate minima. To give an example, the first excited TDHF state in case of $\Omega=5$ stops at $\chi \approx 3$. This reflects the fact that it should merge into the second ground state of the system, see the exact solution for comparison. Thus the dashed line in the figure should thought to be discontinously connected to $\omega_{1}=0$ and this behaviour serves as an approximation to the exact curve, a crude approximation indeed, but at least the behaviour is qualitatively present. Similar considerations apply in all the other cases where a TDHF branch stops. We thus see that quantized TDHF gives a very robust description in all ranges of $\chi$. It is quantitatively correct almost everywhere, except at the discontinuities of the branches. It provides at least the proper qualitative behaviour in those critical cases where branches of solutions disappear or creep up. And, these critical cases can be clearly identified such that one knows where to trust the solution and where better not.

\section{Conclusion}

In this paper, we have investigated two microscopic theories for multiple resonance excitations in nuclei, the RPA- $N$, an extension of the RPA to higher $n$ - $p h$ states, and quantized TDHF. The RPA- $N$ stands for the class of theories which are based on opertor expanions and the Heisenberg equations-of-motion. A close relative to RPA- $N$ are the Boson Expansions such that our findings apply for these as well. The RPA- $N$ is an expansion in orders of excitations which is easiest and most reliable for low excitation modes and becomes increasingly cumbersome with increasing energies and increasing particle number. The quantized TDHF, on the other hand, concentrates on the time-dependent state of the system in the Schrödinger picture. The mean-field approximation, employed in TDHF, corresponds to the classical limit 
of the dynamics of the many-body system. It thus becomes increasingly valid with increasing energy and increasing particle number. From this formal point-of-view, RPA- $N$ and quantized TDHF cover contrary regimes. RPA- $N$ starts an expansion from the lowest excitation modes and stays fully quantum mechanical throughout each step whereas quantized TDHF as a classical approach looks more appropriate for high energies and has to be semiclassically requantized.

The practical comparison has been performed within the Lipkin-Meshkov-Glick (LMG) model which mimics the nuclear excitation dynamics as well as a structural transition from symmetric to deformed ground states. We have studied three typical cases representing a small, a medium, and a heavy nucleus. The RPA- $N$ is found to work fine in the domain of well bound systems, i.e. far below and far above the critical transition point. However, it runs into serious difficulties around the critical point which cannot be surmounted even by high order expansion (actually we have tested it up to $N=5$ ). Quantized TDHF, on the other hand, provides surprisingly robust results for each excitation energy and in each structural domain including the transition point. The classical character of TDHF shines through in the fact that it is somewhat less reliable for the smallest system in our sample. We thus conclude that quantized TDHF is superior in most domains, but RPA- $N$ remains the method of choice for small systems at not too high excitation energies.

The simplicity of the LMG model inhibits practical estimates of the expense of the methods. The RPA- $N$ at full extend will become unfeasible for a realistic nucleus, except perhaps $N=2$ for small nuclei. Higher $N$ can be treated as Boson Expansion with a basis of fixed 1-ph excitation operators. The necessary algebra of multiple commutators can be carried through nowadays but will become very cumbersome. Quantized TDHF, on the other hand, is formally very simple as it employs always as a unique ingredient a TDHF code which is nowadys easily available. But it poses the serious technical problem to find the branches of periodic orbits in the chaotic multitude of solutions. This could turn out to be a big hindrance for the application of the otherwise very straightforward and robust approach.

We would like to thank C. Toepffer for his constant interest on the work and many helpful comments. D.K. and M.W. have been supported by the Studienstiftung des 
Deutschen Volkes.

\section{References}

[1] D.M. Brink, D. Phil. thesis, Oxford University, Unpublished (1955).

[2] J. Ritman et al., Phys. Rev. Lett. 70 (1993) 533.

[3] C. Yannouleas, Phys. Rev. C, 35 (1987) 1159.

[4] D. Beaumel and Ph. Chomaz. Ann. Phys. (N.Y.) 213 (1992) 405.

[5] G. Lauritsch and P.-G. Reinhard, Nucl. Phys. A 509 (1990) 287.

[6] F. Iachello and A. Arima The Interacting Boson Model Cambridge University Press, Cambridge 1987.

[7] A. Klein and E.R. Marshalek, Rev. Mod. Phys. 63 (1991) 375.

[8] P.-G. Reinhard and E.W. Otten, Nucl. Phys. A 420 (1984) 173.

[9] A. Bulgac, Nucl. Phys. A 545 (1992) 545c.

[10] D.J. Rowe, Nuclear Collective Motion, Methuen, London 1970

[11] D.J. Rowe, Nucl. Phys. A 107 (1968) 99.

[12] J.W. Negele, Rev. Mod. Phys. 54 (1982) 913.

[13] H.J. Lipkin, N. Meshkov, and A.J. Glick, Nucl. Phys. 62 (1965) 188.

[14] N. Meshkov, A.J. Glick, and H.J. Lipkin, Nucl. Phys. 62 (1965) 199.

[15] D.J. Thouless, The quantum mechanics of many body systems, Academic Press, New York, 1961.

[16] P. Ring, P. Schuck, The Nuclear Many-Body Problem, Springer, Ney York 1980.

[17] S. Levit, J.W. Negele, and Z. Paltiel, Phys. Rev. C 21 (1980) 1603. 
[18] J.P. Blaizot, H. Orland, Phys. Rev. C 24 (1981) 1740.

[19] H. Reinhardt, Nucl. Phys. A 369 (1981) 109.

[20] K.-K. Kan, J.J. Griffin, P.C. Lichtner, and M. Dworzecka, Nucl. Phys. A 332 (1979) 109.

[21] K.-K. Kan, Phys.Rev. C 22 (1980) 2228.

[22] M. Baranger, Ann. Phys. (N.Y.) 186 (1988) 95.

[23] G. Holzwarth, Nucl. Phys. A 207 (1973) 545. 
Figure 1:

Visualization of the deformation energy (solid lines) versus the collective deformation angle $\psi$ for the LMG model with $\Omega=14$ particles at three different coupling strengths as indicated. The energy of the first excited mode is indicated by a dashed line in each case.

Figure 2:

Spectra of the LMG model as a function of the strength of the residual interaction in the range of undercritical coupling $\chi<1$ for three particle numbers $\Omega$ as indicated. The different line types represent different approximation schemes: solid = exact solution, dashed $=$ quantized TDHF, dash-dotted $=R P A-5$, dotted $=R P A-2$.

Figure 3:

Spectra of the LMG model as a function of the strength of the residual interaction in a broad range of couplings for three particles number $\Omega$ as indicated. The different line types represent different approximation schemes: solid = exact solution, dashed $=$ quantized TDHF, dash-dotted $=R P A-5$, 'spherical' ground state and RPA-2, 'deformed' ground states, dotted $=R P A-2$, 'spherical' ground state. Note that a RPA-5 on a 'spherical' ground state and a RPA-2, 'deformed' ground states both describe the first six eigenstates of the Hamiltonian. 